\title{
STABILITY AND STRUCTURE OF INVERSE SWIRL DIFFUSION FLAMES WITH WEAK TO STRONG SWIRL
}

\author{
A.M. Elbaz ${ }^{\mathrm{a}}$ *, and W.L. Roberts ${ }^{\mathrm{a}}$ \\ *ayman.elhagrasy@kaust.edu.sa \\ ${ }^{a}$ Clean Combustion Research Center, King Abdullah University of Science and Technology, KAUST, Saudi \\ Arabia
}

\begin{abstract}
Flame stabilization in many practical devices is achieved primarily through swirl; however, the application of swirl to an inverse diffusion flame and its effect on both flame stability and structure have not yet been reported. Therefore in this work, flame stability and structure of inverse diffusion swirling flames are investigated. The most stable flame is achievable at a critical swirl intensity, $S_{\mathrm{cr}}$. Lifted swirl jet-like flames at swirl intensity $<S_{\mathrm{cr}}$, and compact flames at swirl intensity $>\mathrm{S}_{\mathrm{cr}}$ are observed. Simultaneous PIV/OH-PLIF measurements are conducted to investigate the flame-flow interaction for three flames. The flame at $S_{c r}$ is stabilized due to the mutual dependence of two flame zones, the conical flame and the flame root. The conical flame zone is located along with the inner shear layer (ISL) between the internal recirculation zone and the jet flow, while the flame root is positioned close to the burner nozzle. The ISL is favorable for the flame to stabilize due to the enhancement of the burned gas and fresh-fuel/air mixing associated with the frequently-formed vortices. The flame root is stabilized due to the instantaneous opposed flow between the hot burned gas and the fresh reactants. The flame root essentially requires the upstream flow of hot exhaust gases associated with the IRZ. It is inherently unstable at the high swirl intensity due to the high strain rate coupled with the strong IRZ. At low swirl intensity, the flame root is extinguished by the high momentum of the central jet, and a lifted flame is anchored in low-velocity regions of the jet, with the axial and radial movement of the flame to meet this criterion. These results emphasize the crucial role of the flame root and suggest that well-aimed
\end{abstract}


modifications of flow field or mixing in this region may increase the stability of the lifted jetlike swirling flames.

\section{Keywords:}

Turbulent Combustion, Inverse swirling flame, Flame stability, Flame structure.

\section{Nomenclature}

IRZ Internal recirculation zone

ISL Inner shear layer

$\mathrm{r} \quad$ Radial distance, $\mathrm{mm}$

S Geometric swirl number

U Bulk air velocity at the burner exit tip, $\mathrm{m} / \mathrm{s}$

V Mean axial velocity, $\mathrm{m} / \mathrm{s}$

Vrms Axial velocity $\mathrm{rms}, \mathrm{m} / \mathrm{s}$

W Tangent velocity component, $\mathrm{m} / \mathrm{s}$

$\mathrm{Z} \quad$ Vertical distance downstream the burner exit, $\mathrm{mm}$

$\Phi \quad$ Global flame equivalence ratio

\section{Introduction}

Relative to premixed flames, non-premixed flames are desirable in practical combustion applications due to the wide range of flame stability and controllability [1]. However, the emissions of soot and $\mathrm{NO}_{\mathrm{x}}$ can be issued with diffusion flames that must be adequately addressed. Lean premixed flames have the advantage of low temperature, and consequently low thermal $\mathrm{NO}_{\mathrm{x}}$ emissions, however, often possess stability limitations. The inverse diffusion flame, IDF, in which a central air jet is admitted into an outer co-axial fuel jet, has broad flame stability, the operational safety inherent in non-premixed flames, and the low emissions typical of premixed flames. These advantages have motivated different research groups to investigate the characteristics of inverse diffusion flames [2-8]. The co-axial air-fuel 
jets geometry is the more common burner configuration of IDF flames studies [2-5]. By including circumferential fuel ports, the emissions and flame appearance relative to the coaxial IDF are significantly altered, as reported in [6].

Several investigations in the literature have been reported on laminar air inverse diffusion flames, IDFs. The stability map of methane-air inverse diffusion flame was investigated in [9]. This was followed by a primary focus on the soot and carbon monoxide formation in the IDFs [10]. Recently, a comparative study of the flame structure of the inverse and normal oxy-fuel diffusion flame for the oxy-fuel arrangement using a combined experimental and numerical approach was reported in [8]. The flame structure of turbulent inverse diffusion flames was investigated by Elbaz and Roberts [2-4] using high-speed OH-PLIF/PIV. In their work, the local flame extinction and re-ignition, together with the blow-out behavior of the turbulent IDFs, were identified.

Stricter regulations on the emissions from gas turbines are continually being implemented. Swirling flames in practical applications such as gas turbine combustors or industrial furnaces are widely used owing to their wider flame stability [11], and the high power density [12]. In contrast to the increasing efforts investigating the IDFs, swirling IDFs have been the subject of fewer studies. Recently, imparting a swirling flow to the air jet in an inverse diffusion flame has been appeared [13-18]. The heat transfer from a turbulent swirling inverse diffusion flame impinging to a flat surface has been investigated experimentally [14]. The comparisons with the normal inverse diffusion flames revealed that the swirling IDF flames have higher combustion efficiency. The heat transfer behaviors of impinging swirling IDFs showed that the addition of swirl to IDFs enhanced the overall heat transfer rate. Additionally, the overall pollutant emission from impinging swirling and non-swirling inverse diffusion flames have been reported in [17]. The effect of the oxygen percentage on the emissions and combustion characteristics of swirling IDFs has also been experimentally studied [18]. The flame 
appearance of swirling IDFs and a non-swirling IDF was compared in [15], where an internal recirculation zone, IRZ, was a dominate the fluid dynamic feature of the swirling IDF. They showed that implementing swirl in IDFs leads to stronger fuel-air mixing, and improves the flame stability relative to non-swirled IDFs [15]. An experimental study was carried out to investigate the thermal and heat transfer behaviors of swirling inverse diffusion flames relative to the non-swirl inverse diffusion flames [13]. They [13] showed some flame appearance differences between the swirl and non-swirl inverse diffusion flames, where the swirl flames showed much shorter flame length. Relative to the premixed swirl flame, Zhen et al. [19] showed that the inverse swirling flames have higher flame stability and minor differences in flame appearance.

The previous literature indicates that implementing swirl to the inverse diffusion flame leads to promising flames in terms of low pollutant emissions and high flame stability. However, the reported data regarding the imparting of swirl to the inverse diffusion flame showed only observations related to the flame appearance, pollutant emissions, and heat transfer. Most of these studies showed that the flame stability is enhanced in the case of swirling IDFs, without reporting the actual flame stability map; they mainly indicate on the flame's appearance. The changing in flame structure as a function of swirl, which is the primary key to explain the reduced emissions and enhanced flame stability, has not been reported. Therefore, the main goal of this contribution is to investigate the stability limits of inverse diffusion flames with swirl, the flame structure, and the flow field. With the aim of a better understanding of the combustion processes in inverse swirling diffusion flames, flame stability regimes are also presented, while the flame/flow structure within the different regions of the stability map is investigated via high-speed OH-PLIF/PIV measurements. 


\section{Experimental setup}

A schematic of the swirl inverse diffusion flame burner is illustrated in Fig. 1a. The exit section of the burner consists of the central air nozzle concentric with an annular slot for the methane supply. The swirl motion is imparted to the central air jet via introducing a tangent air stream through four tangent inlets to the swirl chamber. The axial air stream is added to the chamber through a ceramic honeycomb placed at the bottom of the chamber and is mixed with the tangential air downstream. The swirling air is contracted through a convergent section to the central nozzle of $12 \mathrm{~mm}$ id. The central nozzle section ends with a $45^{\circ}$ divergent exit, where, via the vertical annular slot, admits the fuel mix with the swirling air jet. The divergent exit of $45^{\circ}$ instead of straight exit is intended to enhance flame stability [20]. The existence of the diverging section at the burner exit triggers a large scale of reversal flow, thus higher flame stability relative to the straight exit burner [21]. Even, in partially premixed jet flames, the existence of the quarl at the burner exit creates a side recirculation zone, which adds another stabilization factor to the partially premixed flames [22-25]. The width of the fuel slot is $2 \mathrm{~mm}$ around the circumference of $17 \mathrm{~mm}$ diameter tube, see Fig. 1a. The divergent section is $5 \mathrm{~mm}$ in height downstream of the central air nozzle, the exit plan of the divergent section is taken as a reference, where the vertical axis $\mathrm{Z}=0 \mathrm{~mm}$. The swirl intensity of the air jet is based on geometrical swirl number, defined as the following equation [26]:

$$
S=\frac{G_{\theta}}{G_{a} R}=\frac{\left(D_{0}-D_{t}\right) D_{o}}{2 D_{t}^{2}}\left(\frac{m_{\theta}}{m_{a}+m_{\theta}}\right)^{2}
$$

Where $G_{\theta}$ and $G_{a}$ are the tangential and axial momentum respectively; $R$ is the characteristic burner radius, and $m_{\theta}$ and $m_{a}$ are the tangential and axial airflow rates respectively, $D_{o}$ is the chamber inner diameter, while $D_{t}$ is the tangent tube inner diameter. 


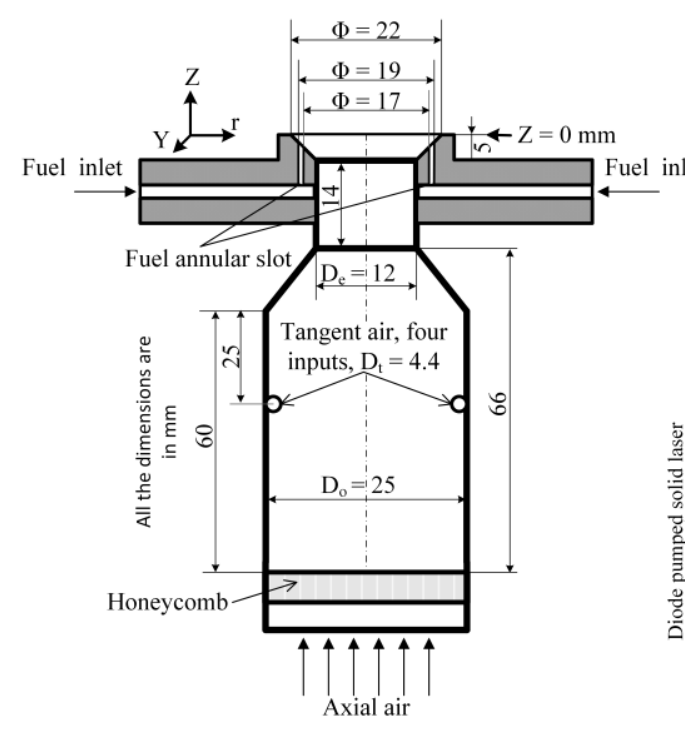

(a)

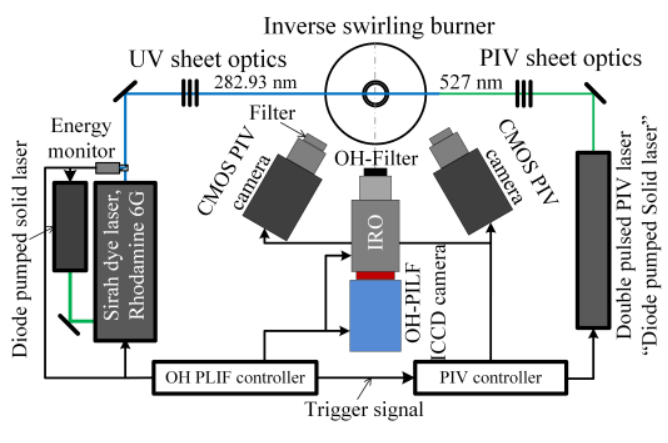

(b)

Fig. 1. (a) Inverse swirling flame burner, (b) Complete experimental apparatus

The experimental arrangement is shown in Fig. 1b. Simultaneous stereoscopic PIV/OHPLIF measurements were conducted for the region just downstream of the burner exit section. The OH-PLIF images were acquired at $10 \mathrm{kHz}$ repetition rate while the simultaneous PIV/OH-PLIF measurements were collected at $1 \mathrm{kHz}$ repetition rate. Two counterpropagating laser beams were formed into sheets using sheet forming optics, focused and overlapped over the vertical central plan of the burner. To illuminate the region of interest for stereoscopic PIV measurements, a dual cavity, diode-pumped, solid-state Nd: YLF laser was used. At $10 \mathrm{kHz}$, the laser was able to produce $35 \mathrm{~mJ} /$ pulse of $527 \mathrm{~nm}$ light, with a $9 \mathrm{~ns}$ pulse duration. Two high-speed CMOS cameras (LaVision, Image Pro HS 4M, HSS5), were used to capture the PIV images. These two PIV cameras were located at $\pm 15^{\circ}$ inclination angle at equidistance from the centerline of the burner on both sides of the OH-PLIF ICCD camera. The images were acquired in two-frame burst mode with a resolution of $720 \times 670$ pixels. The Mie-scattered light from the seeding particles laden the flow was captured via $105 \mathrm{~mm}, \mathrm{f} / 4$ objective lens, with a $527 \mathrm{~nm}$ bandpass filter fitted to the lens inlet. The perspective distortion 
in the camera images was corrected and calibrated by using a dual plane, three-dimensional calibration target, LaVision type 22. The raw images were processed using the LaVision Davis 8.1 software package, where first the image de-warping between the stereoscopic cameras was corrected via image correction and distortion function. The system coordinate and camera calibration were adjusted so that the $\mathrm{Y}=0 \mathrm{~mm}$ plane (see Fig. 1a) occurred in the middle of the laser sheet through the self-calibration function. The velocity vectors were calculated by applying particle image spatial cross-correlation, where an adaptive multi-pass vector evaluation algorithm was used (the interrogations areas changed from 128 pixels to 16 pixels). With $16 \times 16$ pixels interrogation windows, $50 \%$ overlap, and the laser sheet of nearly 1-mm waist, a spatial resolution of approximately $1.2 \mathrm{~mm} \times 1.2 \mathrm{~mm} \times 1 \mathrm{~mm}$ was achievable. Both the swirling and fuel jets were seeded with titanium dioxide particles of $0.5 \mu \mathrm{m}$ nominal diameter. With $0.5 \mu \mathrm{m}$ particle seeding diameter, the cut-off frequency in the turbulent flow is estimated to be $60 \mathrm{kHz}[12]$.

For OH-PLIF measurements, a frequency-doubled high-speed dye laser from Sirah (Cerdo-Dye) pumped with a frequency-doubled, diode-pumped solid-state INNOSLAB laser from Edgewave (IS16II-E) was used to generate UV light. With Rhodamine 6G, the dye laser produced a fundamental beam at $566 \mathrm{~nm}$, which is doubled by using a BBO crystal to produce a $283 \mathrm{~nm}$ laser beam at a $10 \mathrm{kHz}$ repetition rate. The average UV laser power was $2.8 \mathrm{~W}$. The UV laser beam with expanded to collimated sheets of approximately $100 \mathrm{~mm}$ height using two cylindrical lenses and focused on at the burner centerline using a third cylindrical lens to about $130 \mu \mathrm{m}$ thickness. The dye laser was tuned to $283.93 \mathrm{~nm}$ to excite the line Q1 (6) in the $\mathrm{A}^{2} \sum+-\mathrm{X}^{2} \mathrm{II}(1,0)$ band. The $\mathrm{OH}$ fluorescence signal was acquired at the right angle to the laser sheet with a high-speed-star 8 CMOS camera (LaVision HSS8) and an external twostage lens coupled intensifier (LaVision, HS-IRO) with an array of $768 \times 888$ pixels. The ICCD camera is equipped with Nikkor UV lenses $(\mathrm{f} / 4.5, \mathrm{f}=105 \mathrm{~mm})$ together with an 
appropriate bandpass interference filter, with a transmission range from $295 \mathrm{~nm}$ to $345 \mathrm{~nm}$, > $80 \%$ at $310 \mathrm{~nm}$, (custom fabrication-laser components $\mathrm{GmbH}$ ). By incorporating laser energy monitor, the shot-to-shot energy variation was corrected to all PLIF images. The effect of the laser beam profile on the row fluorescence images was also corrected using the average ensemble images of 10, 000 images to the laser sheet passing through a cuvette filled with acetone. Also, by averaging 5000 frames while shutting the laser, the background and camera noise was corrected in all the data series.

\section{Results and discussion}

\subsection{Flame stability and selected flames}

The stability results are presented in Fig. 2 as the relationship between the fuel flow rate (methane) on the vertical axis and the geometrical swirl number, S, on the horizontal axis. The flame stability point was achieved by gradually decreasing the fuel flow rate supplied to the burner, while the airflow rates for both air streams (axial and tangential streams) were kept constant until the flame is extinguished. At this point, the fuel flow rate was recorded, and the stability point was defined as the point where the fuel flow rate below which the flame is extinguished. With two air bulk jet velocities, $\mathrm{U}$, of 7.5 and $10 \mathrm{~m} / \mathrm{s}$ (based on the bulk air flow rates and the exit area of the air tube), the flame stability was mapped over a wide range of swirl intensities. The swirl number, $S$, was varied between $S$ of 0 to 12 . At $S=0$, the airflow was only introduced through the axial inlet to the burner, and inverse diffusion flame with no swirl is recognized, IDF. Starting from high swirl number, right side of Fig. 2, with a decrease in the swirl intensity, the fuel flow rate for the flame to blow-off was decreased, reaching an optimum swirl intensity which corresponded to the minimum fuel required to maintain stable flame, (referred to hereafter as critical swirl number, $\mathrm{S}_{\mathrm{cr}}$ ). This critical point is slightly shifted towards the high swirl number when increasing the bulk air jet velocity from 7.5 to $10 \mathrm{~m} / \mathrm{s}$. 
With a further decrease in the swirl intensity below $S_{\mathrm{cr}}$, the flames began to detach from the burner tip, showing swirling-jet like flames, (the flame appearance will be illustrated in Fig. 3). More fuel is required to stabilize the flame located in this region of the stability map. At $S=0$, achieving an attached inverse diffusion flame at a relatively low fuel flow rate can be reached. It is also shown in Fig. 2 that the flame at $S_{c r}$ stabilizes at overall equivalence ratio $\sim 0.9$ relatives to the rich mixtures of the IDF with zero swirl intensity (approximately $45 \%$ reduction in the fuel of the zero swirl inverse diffusion flame, i.e. IDFs flames). The blow-off flame limit increases with increasing the jet bulk velocity, as shown for flames at $U=10 \mathrm{~m} / \mathrm{s}$. For $\mathrm{U}=7.5 \mathrm{~m} / \mathrm{s}$, the stability limit at $\mathrm{S}=0$ matched approximately the blow-off limit at $\mathrm{S} \approx 8$ (illustrated by the red dashed line), a further increase in $\mathrm{S}$ lead to a rise in the fuel flow rate, i.e., increasing the flame blow-off limit. However, for $\mathrm{S}$ from 0 to 12 , at the higher bulk velocity at $\mathrm{U}=10 \mathrm{~m} / \mathrm{s}$, imparting swirl on the flow improved the flame stability relative to the IDF.

To understand the stability map, and the structure of the inverse diffusion swirling flame, three flames at $U=7.5 \mathrm{~m} / \mathrm{s}$ were selected in this work for detailed measurements. In these three flames, the Reynolds number based on U and air nozzle exit diameter is about 6200 . The flow conditions of these flames and their designations are listed in Table 1, while their positions relative to the blow-off limit are displayed in Fig. 2. The three flames are off from the blow-off limit by $20 \%$ and located into three different swirl intensity zones. One flame is located at the optimum swirl intensity, $S_{\mathrm{cr}}=2$, and the other two flames are located to the right and the left of this flame on the stability plot. This means imparting swirl to the IDFs leads to the improvement of the flame stability within a specific window of swirl intensity, which is bounded by the red dotted lines for $U=7.5 \mathrm{~m} / \mathrm{s}$ bulk air velocity in Fig. 2 . 


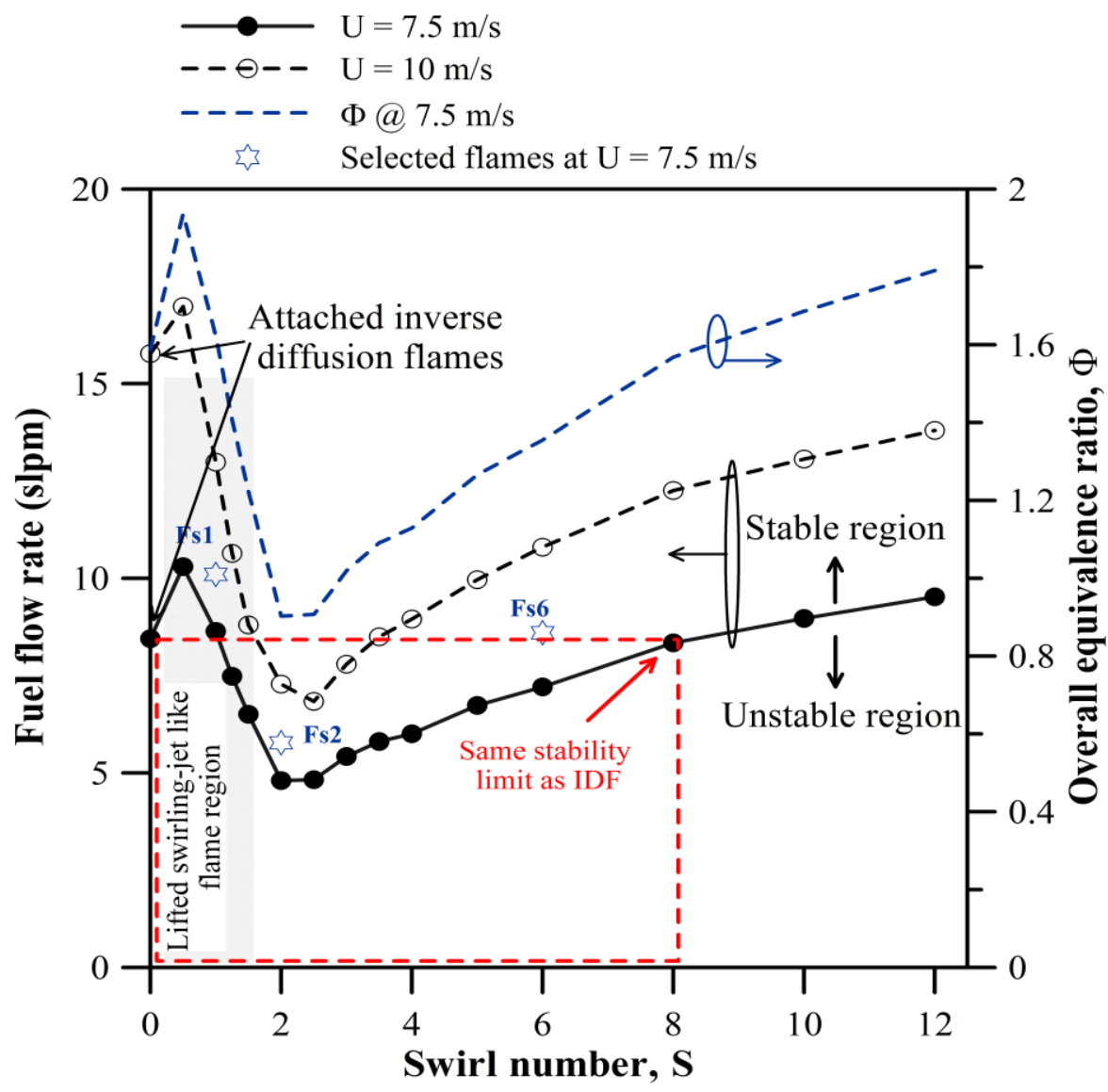

Fig. 2. Inverse swirling flame stability map at two $U=7.5$, and $10 \mathrm{~m} / \mathrm{s}$

\subsection{Average characteristics of the flames}

Figure 3a shows the mean $\mathrm{OH}^{*}$ chemiluminescence (electronically excited) images of the flames Fs1, Fs2, and Fs6. In each image, the $\mathrm{OH}^{*}$ signal is normalized by the maximum signal intensity. The $\mathrm{OH}^{*}$ images indicate the flame shape and act as a marker of the heat release zone [27]. When chemiluminescence is compared to LIF (which samples the electronic ground state of target species), it offers qualitative information about the concentration of species produced in the excited electronic stat from equilibrium. The near UV spontaneous emission from $\mathrm{OH}^{*}$ is visible only in the heat release zone and not in the region of burned gas. $\mathrm{OH}^{*}$ is a line of sight methodelogy; the spatial resolution is compromised. For more clarity, we presented the deconvoluted chemilumiescscence image obtained from the time-averaged image in Fig. 3b. Form both Figs 3a-3b, for flame Fs1, the heat release zone starts at $\mathrm{Z}=8 \mathrm{~mm}$ in the axial direction and localized in the annular shear 
layer of the jet flow $(r= \pm 10 \mathrm{~mm})$, indicating that the flame is not attached to the exit nozzle. However, further downstream, the heat release zone is diverged and distributed radially. Flame Fs2 shows a short compact flame, and the heat release zone lies between $\mathrm{Z}=1$ and 35 $\mathrm{mm}$ and within a radius of $\mathrm{r}< \pm 25 \mathrm{~mm}$. When the swirl number is further increased to 6 , flame Fs6, the flame tip is extended to $\mathrm{Z} \approx 50 \mathrm{~mm}$, and the peak $\mathrm{OH}^{*}$ signal is radially shifted compared to flame Fs2. A significantly dimmer signal within the central flame region is observed in flame Fs6. Flame Fs6 also shows a slight lift-off from the exit nozzle of approximately $2 \mathrm{~mm}$. As will be indicated later, this is attributed to the strong recirculated flow associated with the inner recirculation zone, IRZ.
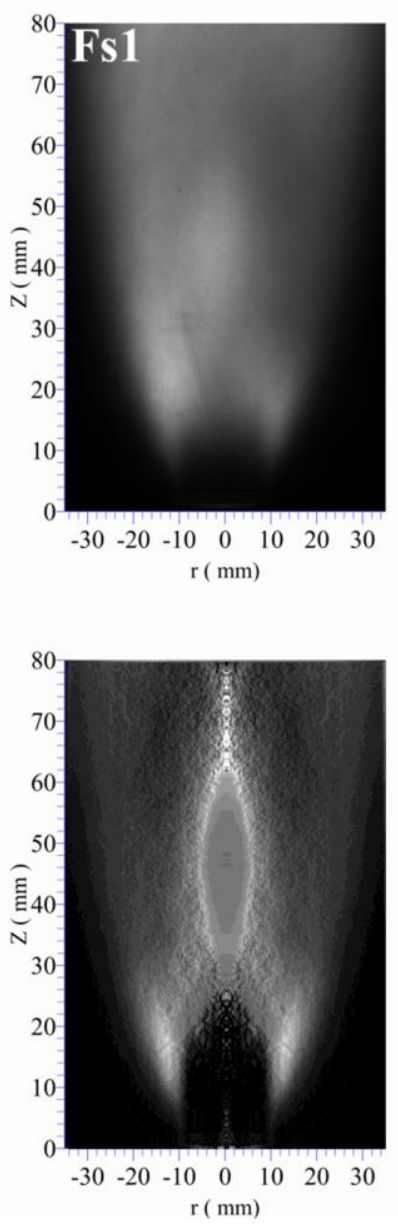

(a)

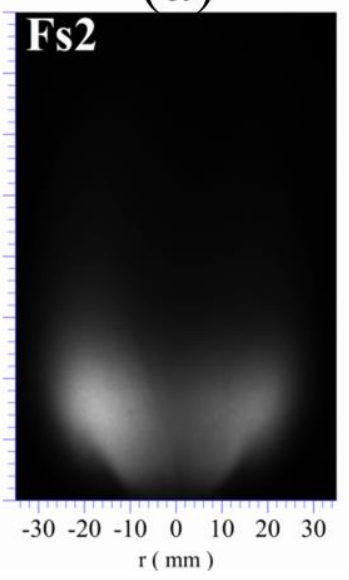

(b)

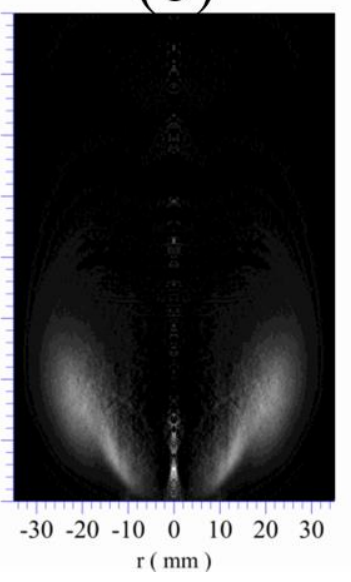

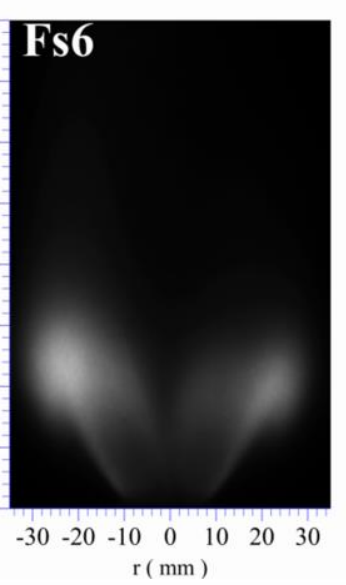

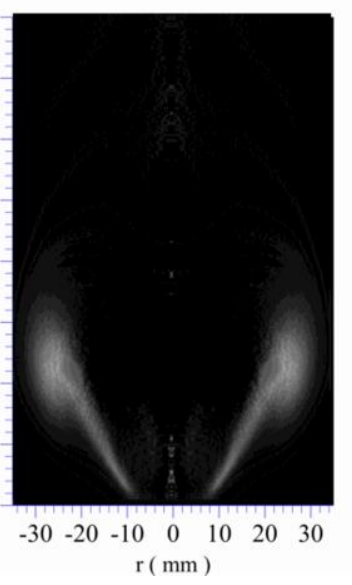

Fig. 3. Average $\mathrm{OH}^{*}$ of the three flames Fs1, Fs2, and Fs6 in (a), and (b) the corresponding deconvoluted $\mathrm{OH}^{*}$ chemiluminescence images 
Table 1. The main parameters of the flames under investigation

\begin{tabular}{|c|l|l|l|l|l|}
\hline Bulk jet velocity, $\mathrm{U}$ & $\mathrm{S}$ & $\mathrm{Q}_{\mathrm{t}}(\mathrm{slpm})$ & $\mathrm{Q}_{\mathrm{a}}(\mathrm{slpm})$ & $\mathrm{Q}_{\mathrm{f}}(\mathrm{slpm})$ & Flame \\
\hline \multirow{3}{*}{$7.5(\mathrm{~m} / \mathrm{s})$} & 1 & 14.6 & 36.4 & 10.3 & Fs1 \\
\cline { 2 - 6 } & 2 & 20.7 & 30.3 & 5.8 & Fs2 \\
\cline { 2 - 6 } & 6 & 35.8 & 15.2 & 8.6 & Fs6 \\
\hline
\end{tabular}

The ensemble-averaged velocity vectors of 2000 stereo PIV recordings for the three flames Fs2 and Fs1-Fs6 are shown in Fig. 4 and Fig. 5, respectively. The measurements covered the entire region of the flame, from the burner exit to $100 \mathrm{~mm}$ downstream. The velocity vector values in the measurement plane are represented by the arrows and are superimposed on the vertical velocity contours, V. The flow at the burner exit swirled clockwise about the central vertical axis, $\mathrm{Z}$, as seen from the top. The flow field features are typical of the unconfined symmetrical swirl flames. From the central nozzle at the bottom, the air is issuing in the form of a cone-shaped stream, referred to hereafter as the 'Jet,' while the fresh fuel issues from an annular slot surrounding the air swirling jet. The air jet is accompanied by a central vortex breakdown that is created by the high tangential momentum. The vortex breakdown generates a central toroidal recirculation zone, IRZ, (Fig. 4a). Part of the flow proceeds back towards the burner nozzle through IRZ; see the zoomed window shown in Fig. 4b. The streamlines in Fig. 4c illustrates the boundaries of the recirculation zone by the dotted red line, where the recirculation zone is defined as the region of the axial velocity is less than zero $(\mathrm{V}<0)$. The reversed flow region occupies most of the space at the burner exit tip. It extends radially to approximately $\mathrm{r} \approx 11 \mathrm{~mm}$ and in downstream distances to $\mathrm{Z}=35 \mathrm{~mm}$ where it ends by an average high stagnation point, hsp, see Figs. 4b-c. One can notice that the IRZ extends back upstream into the air nozzle; thus, the average lower stagnation point is located upstream the measurement field of view, $\mathrm{Z}<0 \mathrm{~mm}$. Due to the IRZ, the incoming jet flow is pushed radially away from the central region; therefore, a strong velocity gradient occurs in the inner shear layer (ISL) between the incoming jet and the IRZ. 
The fuel from the annular fuel slot mixes immediately with the swirling air due to the early formation of the IRZ. This promotes fuel/air mixing and reaction rates; therefore, the flame surface is most probably located in this region and provides a continuous source of heat and radicals to the incoming fresh mixture. An outer shear layer, OSL, is formed between the inflow jet and the surrounding environment.

Figure 5 illustrates the zoomed windows of the near velocity vectors fields and streamlines of flames Fs6 and Fs1. With a further increase of the swirl intensity, as in flame Fs6, Fig 5a-b, the features of the mean flow field appear reasonably similar to those described features for flame Fs2, illustrated in Fig. 4. However, with a close inspection of the velocity vectors close to the burner exit, Fig. 5a indicates some quantitative differences. The flow field is dominated by a larger inner recirculation zone IRZ as depicted in Fig. 5b. The IRZ is flanked by a faster but thinner annular flow jet at the burner exit, thus, a stronger inner shear, ISL. The high swirl intensity leads to the outward radial expansion of the swirling jet as indicated by the streamlines shown in Fig. 5b, thus, a larger size of the toroidal vortex and the movement of the vortex eye more downstream relative to flame Fs2. It is evident that the average high stagnation point on the flame centerline is pushed further downstream in flame Fs6. A part of the outer recirculation zone, ORZ, is seen at the outer shear layer of the inflow jet and located close to the burner exit. This leads to surrounding air entrainment to the early part of the flame. The flow field of the flames located within the lifted swirl like jet flame region of the stability map, Fig. 2, shows distinct differences in the flow field to those flames placed on the swirling attached flame region. As shown in Fig. 5c-d the near flow field of flame Fs1 indicates the structure of jet flow with a peak jet velocity lies in the central region, with no formation of an IRZ. The flow shows a significant decrease in radial jet spreading relative to flames Fs2 and Fs6. However, due to the detachment from the burner nozzle, as illustrated in Fig. 3, the velocity vectors at the jet shear layer at $\mathrm{Z} \approx 15 \mathrm{~mm}$ shows a slightly 
outward direction leading to radial jet expansion. This outward velocity vector ceases at further downstream distances, as indicated by the velocity vector fields and streamlines in Fig. 5c-d.
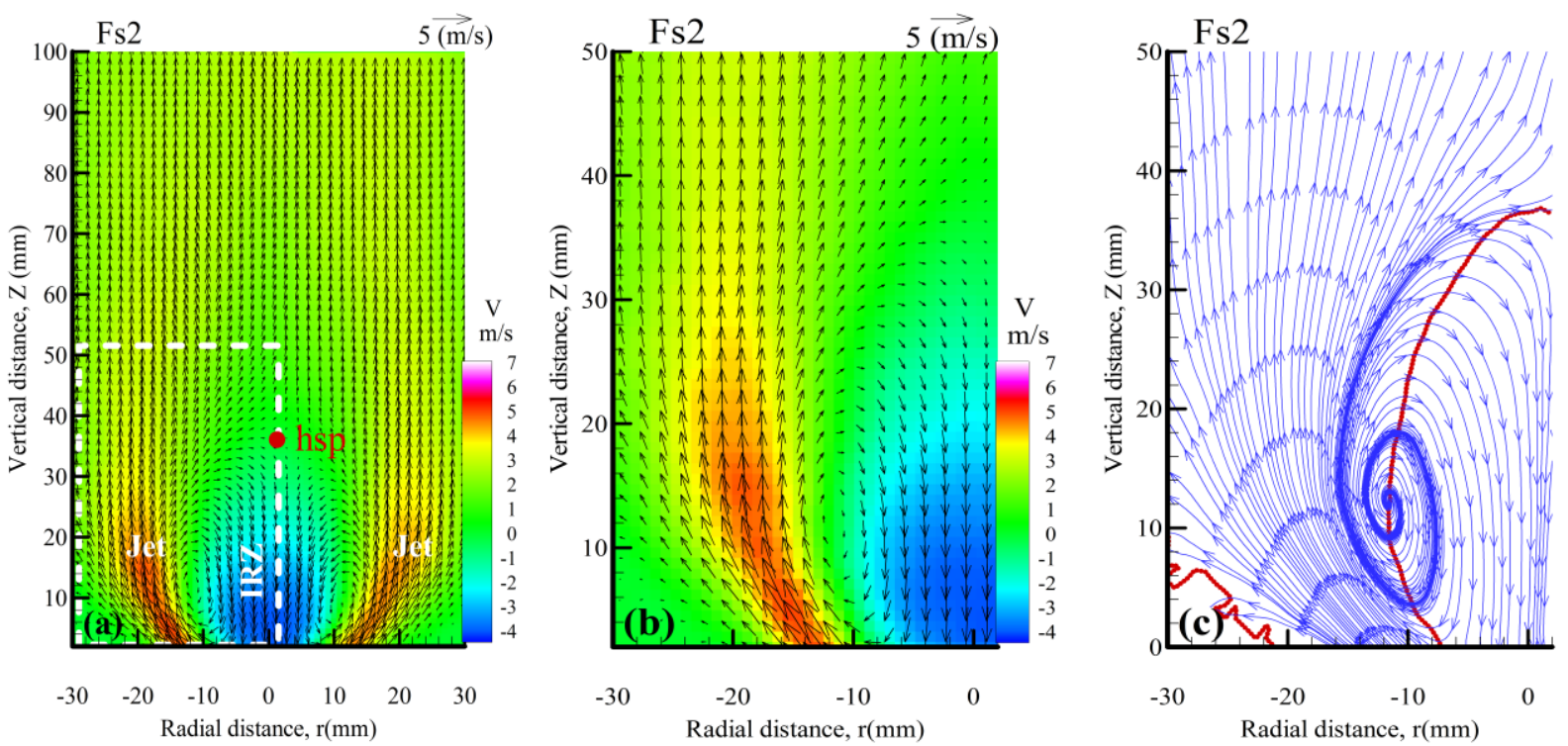

Fig. 4. Time average flow field of flame Fs2: (a) velocity vectors overlaid the contour plot of vertical velocity components $(\mathrm{V})$, (b) zoomed flow field of the dashed white bounded region shown in (a), alternate rows of vectors have been removed for clarity, and the corresponding streamline plot is shown in (c), the dotted red line represents the boundaries of the reversed flow region.
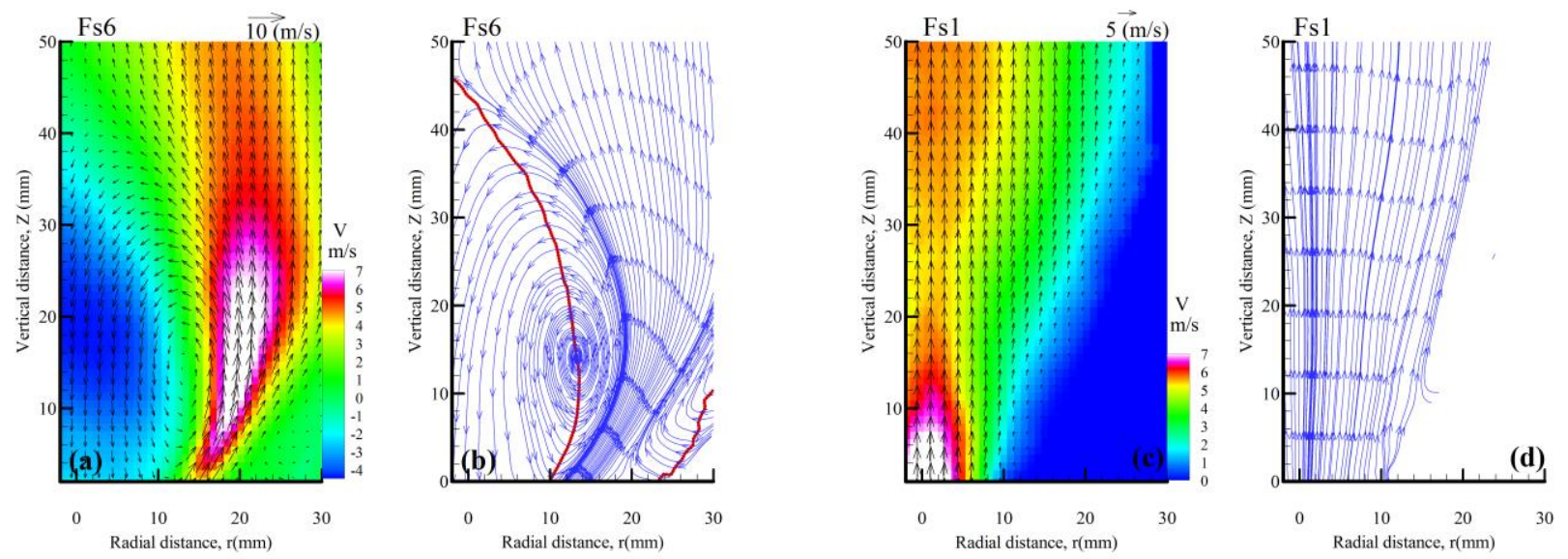

Fig. 5. Time average flow field of the early right region of flames Fs6 and Fs1, for flame Fs6 (a) velocity vectors overlaid the contour plot of vertical velocity components $\mathrm{V}$, (b) the corresponding streamline plots is shown in (b), the plots of Fs1 are shown in (c) and (d) 
To further investigate the features of these three different flame flow fields, the radial profiles of the mean axial velocity, V, and the velocity fluctuations, Vrms, at various axial locations in the three flames are illustrated in Fig. 6. The velocity profiles at the near-axial distances within the lifted flame zone of flame Fs1, $\mathrm{Z} \leq 20 \mathrm{~mm}$, Fig. $6 \mathrm{a}$, show the typically developed jet profiles with a central peak velocity. The corresponding Vrms profiles at $Z \leq 20$ mm, Fig. 6d, exhibit peaks positioned at the outer jet shear layer and corresponding to the steep velocity gradient. As shown, the Vrms peaks are gradually decreased downstream. At Z $\geq 30 \mathrm{~mm}$, the heat release accompanied by the flame leads to thermal expansion and prevents a decrease of the maximum mean jet velocity, regardless of the radial jet expansion. Thus, the central region of the flame maintains a nearly uniform profile and damped Vrms levels for long downstream distances, and this is also seen in $\mathrm{OH}^{*}$ image, Fig. 3.

The prominent features of the profiles of the axial velocity for flame Fs2 are the inflow regions jet with a peak velocity of nearly $5.4 \mathrm{~m} / \mathrm{s}$ and pronounced IRZ with a maximum negative velocity of $4 \mathrm{~m} / \mathrm{s}$ along the flame centreline for $Z=5 \mathrm{~mm}$, as illustrated in Fig. $6 \mathrm{~b}$. Up to nearly $Z=20 \mathrm{~mm}$, the local maxima of the inflow jets are gradually decreased and shifted outwards; this is associated with a progressive decrease of the negative velocity within the IRZ. Beyond $Z=35 \mathrm{~mm}$ at the end of IRZ, the peak velocities of the annular jet start to converge toward the central region. The Vrms profiles of Vrms in Fig. 6e show only local maxima on the inner shear layer of the inflow jet, and minimum Vrms values within the inner region of the flames. For $\mathrm{Z}<40 \mathrm{~mm}$, the radial locations of the peak Vrms follow the velocity profiles and show an outward shift associated with a broadening of the profiles.

On the other hand, the velocity profiles of flame Fs6 depicted in Fig. 6c shows a sharp velocity increase in the vicinity of the IRZ close to the burner exit. This is attributed to the broader recirculation zone penetrating the upstream air tube. The reversed flow regions showed a different variation than those examined for flame Fs2, where they indicate a nearly 
uniform profile $(\approx 3 \mathrm{~m} / \mathrm{s}$ ) close to the burner exit at $Z=5 \mathrm{~mm}$. Further downstream, the reversed velocity increases, reaching a maximum value of around $5 \mathrm{~m} / \mathrm{s}$ at $\mathrm{Z}=20 \mathrm{~mm}$. This indicates the downstream shift of IRZ's eye with increasing the swirl intensity, as well as wider and elongated IRZ. At further axial distances, the reversed flow starts to decelerate where the high stagnation point is located, around $Z=45 \mathrm{~mm}$, see Fig. 5. Relative to those of flame Fs2, the Vrms profiles close to the burner exit of flames Fs6 illustrated in Fig. 6f shows a sharper profile and a relatively higher peaks Vrms within the inner shear layer of inflow jet. This sharp Vrms profiles turned into a gradual decrease from higher values at the shear layer to relatively lower values within the IRZ, which shows a plateau of low Vrms.

As expected with the high $\mathrm{S}$, the tangential velocity component, $\mathrm{W}$, is conserved for a longer downstream distance than those of low S, as illustrated by the radial profiles of the swirling velocity at $Z=40 \mathrm{~mm}$ in Fig. 7. It depicts the steepest profile across the central region for flame Fs6. This explains the longer extent of the IRZ in flame Fs6. One can notice that there is still low tangent velocity in flame Fs1, which was not able to create vortex breakdown and thus no recirculating flow. 

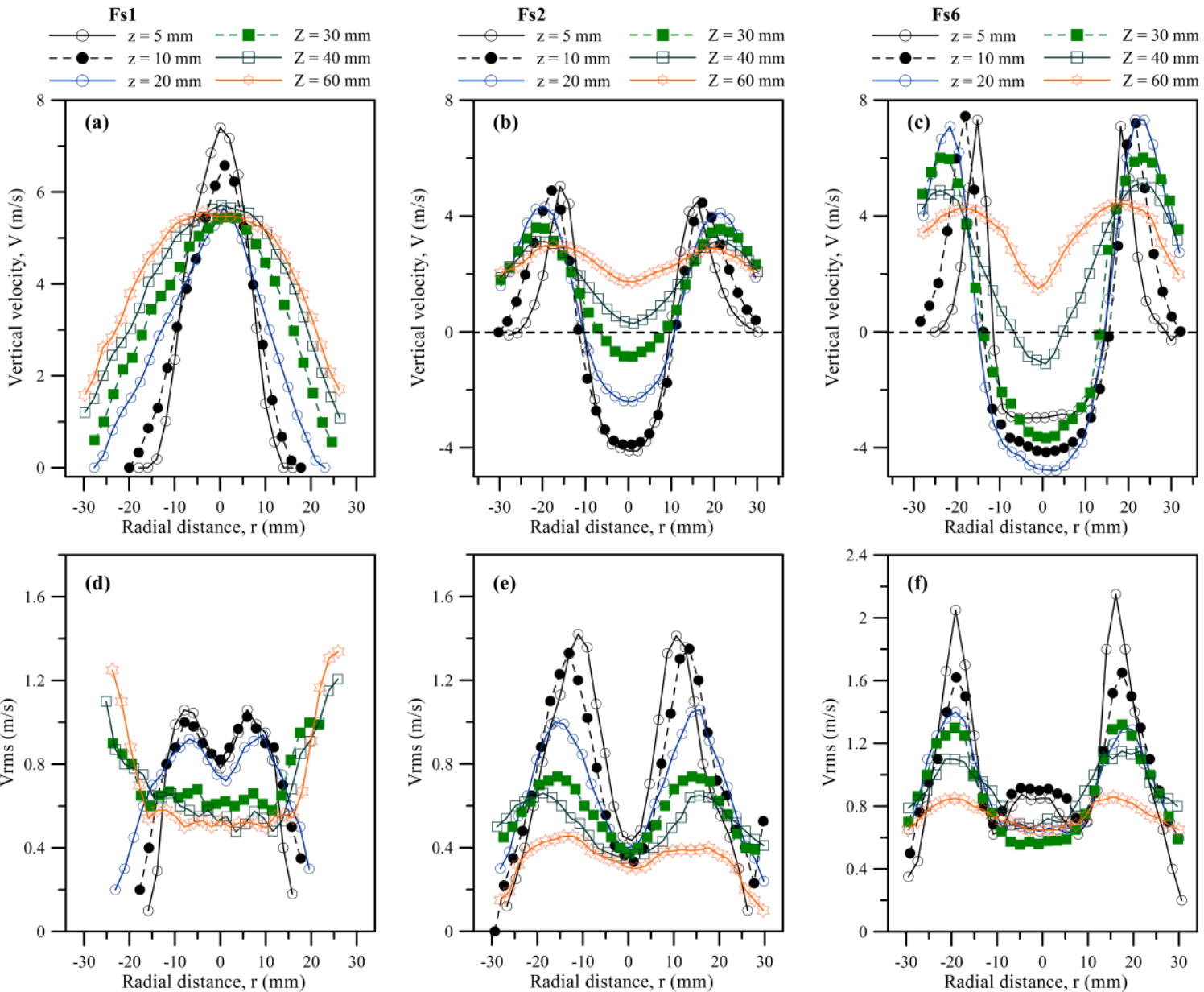

Fig. 6. Radial plots of mean axial velocity at different axial locations of flames: (a) Fs1, (b) Fs2, and (c) Fs6 (top row) in the bottom row the corresponding axial rms velocity fluctuations in (c), (d) and (f), respectively.

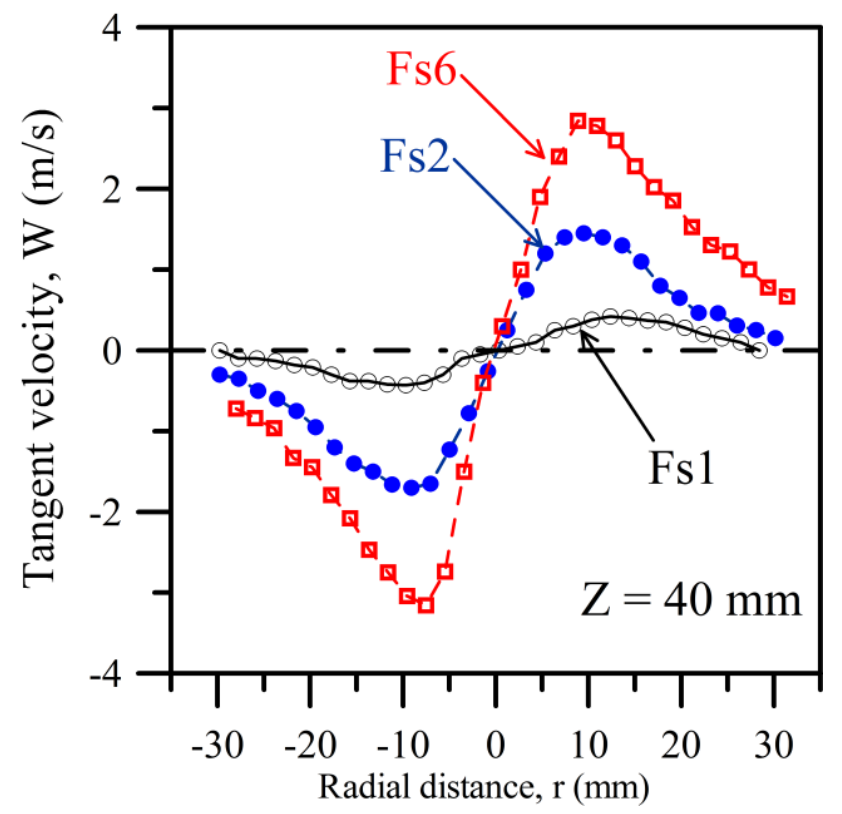

Fig. 7. Profiles of the tangent velocity components, $\mathrm{W}$, at $\mathrm{Z}=40 \mathrm{~mm}$ of the three flames. 


\subsection{Instantaneous flame structure and flow field}

The presented averaged flow field provides insights into the primary fluid dynamic features of the flame, while the instantaneous flow structures are significant in understanding the flame stability, mixing field, and blow-off behavior. Samples of the instantaneous streamlines for the three investigated flames are presented in Fig. 8. It is evident that instantaneous flow fields of flames Fs2, and Fs6 show dramatically different features from the mean flow field (presented in Fig. 4 and Fig. 6), where strong temporal fluctuations of the structures occur. For flames Fs2 and Fs6, the flow fields indicate the existence of multiple small-scale vortical structures in the region close to the ISL, and that is in contrast to the mean flow field, which contains sizeable internal recirculation zone. These small vortices are continuously formed and dissipated in the shear layer. The flow field of flame Fs1, Fig. 8c does not show these vortical structures. The centers of the vortices associated with flame Fs2, Fig. 8a, are located close to the burner exit, while those of flame Fs6, Fig. 8b, are found further downstream the burner exit and radially shifted from the central region. Thus, early and intense mixing between the cold fresh mixtures and hot burned gases from the IRZ in flame Fs2 exists.

It is useful to study the evolution of the flame structure by investigating the high-speed OH-PLIF $(10 \mathrm{kHz})$ images. The flame flow interaction is examined through the inspection of the simultaneous OH-PLIF/PIV measurements conducted at $1 \mathrm{kHz}$. Figure 9a shows a typical time-sequence of OH-PLIF images of flame Fs2 over a time interval of $2 \mathrm{~ms}$ ( 7 frames) to match the PIV frame rate for the three of the presented OH-PLIF images. The simultaneous PIV velocity vectors and OH-PLIF are displayed in Fig. 9b (for the first, fourth, and the seventh $\mathrm{OH}$ frames). Close inspection of the $\mathrm{OH}$ images identifies different flame zones, (see Fig. 9a). High $\mathrm{OH}$ levels confirm that super-equilibrium levels exist in the reaction zones [28]. Medium to low levels of $\mathrm{OH}$ signals indicate burned gas where the $\mathrm{OH}$ levels decay toward equilibrium while being transported away from the reaction zone in the post-flame 
regions. Dark regions where there is no $\mathrm{OH}$ signal show the low-temperature gases (fresh gases or diluted burned gases). To identify the threshold level of the $\mathrm{OH}$ concentration representing the superequilibrium, i.e., $\mathrm{OH}$ that formed in a reaction zone or moved a short distance from the reaction zone, a PREMIX code available in Chemkin-Pro was performed using GRI3.0 mechanism. In the current fame configuration, fuel and air are not premixed to the global equivalence ratio, so the reactions are most likely to occur at $\Phi \approx 1$. However, due to the internal recirculation zone generated within the central region of the flame, which may lead to the change in $\Phi$ from stoichiometric, thus the $\mathrm{OH}$ mole fraction at $\Phi=0.75$ was also calculated. At $\Phi=1$, the results show that the maximum $\mathrm{OH}$ mole fraction is 2.5 times the equilibrium concentration, while at $\Phi=0.75$ is around four times the equilibrium $\mathrm{OH}$ concentration. Accordingly the threshold $\mathrm{OH}$ level of the super-equilibrium was defined and is presented on the color legend bar of the $\mathrm{OH}$ concentration in Fig. 9 (by the horizontal white arrow). The same methodology has been used by Sadanandan et al. [29]; however, they took into account the effect of the diluted gases as an input in their simulation, and they did not observe a significant effect on the level of $\mathrm{OH}$ concentration. The time sequences of the instantaneous OH-PLIF images of flame Fs2 exhibit many essential characteristics. 

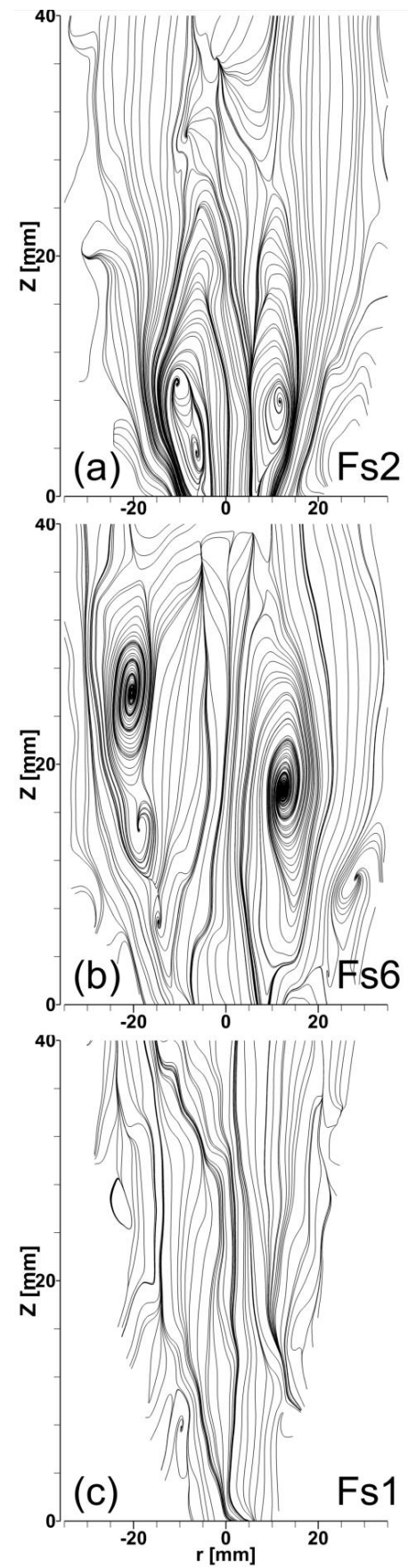

Fig. 8. Instantaneous streamlines plots: (a) flame Fs2, (b) flame Fs6, and (c) flame Fs1 

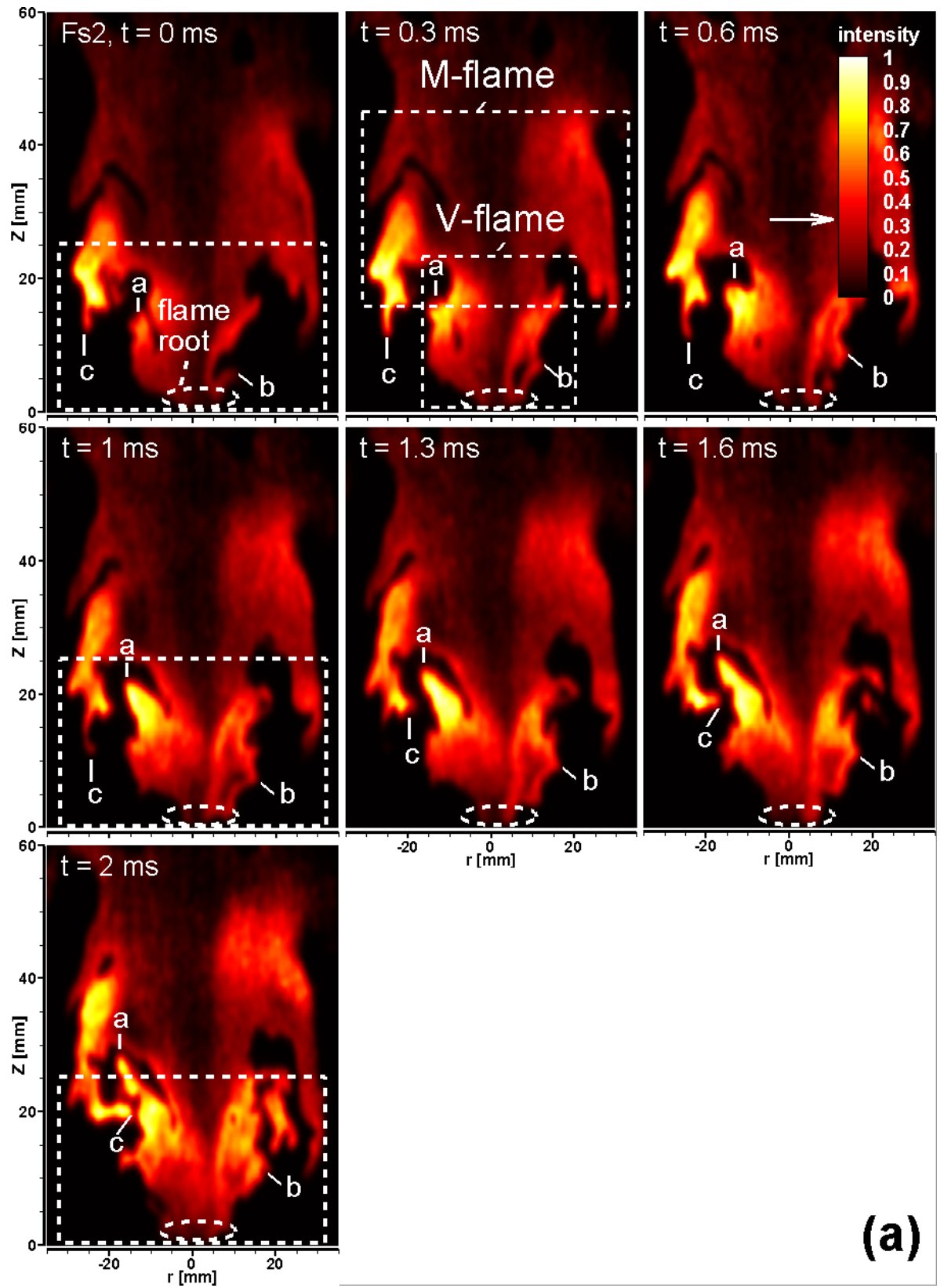

(a)

Fig. 9. Flame Fs2: (a) Time sequence of the OH-PLIF of flame Fs2. 


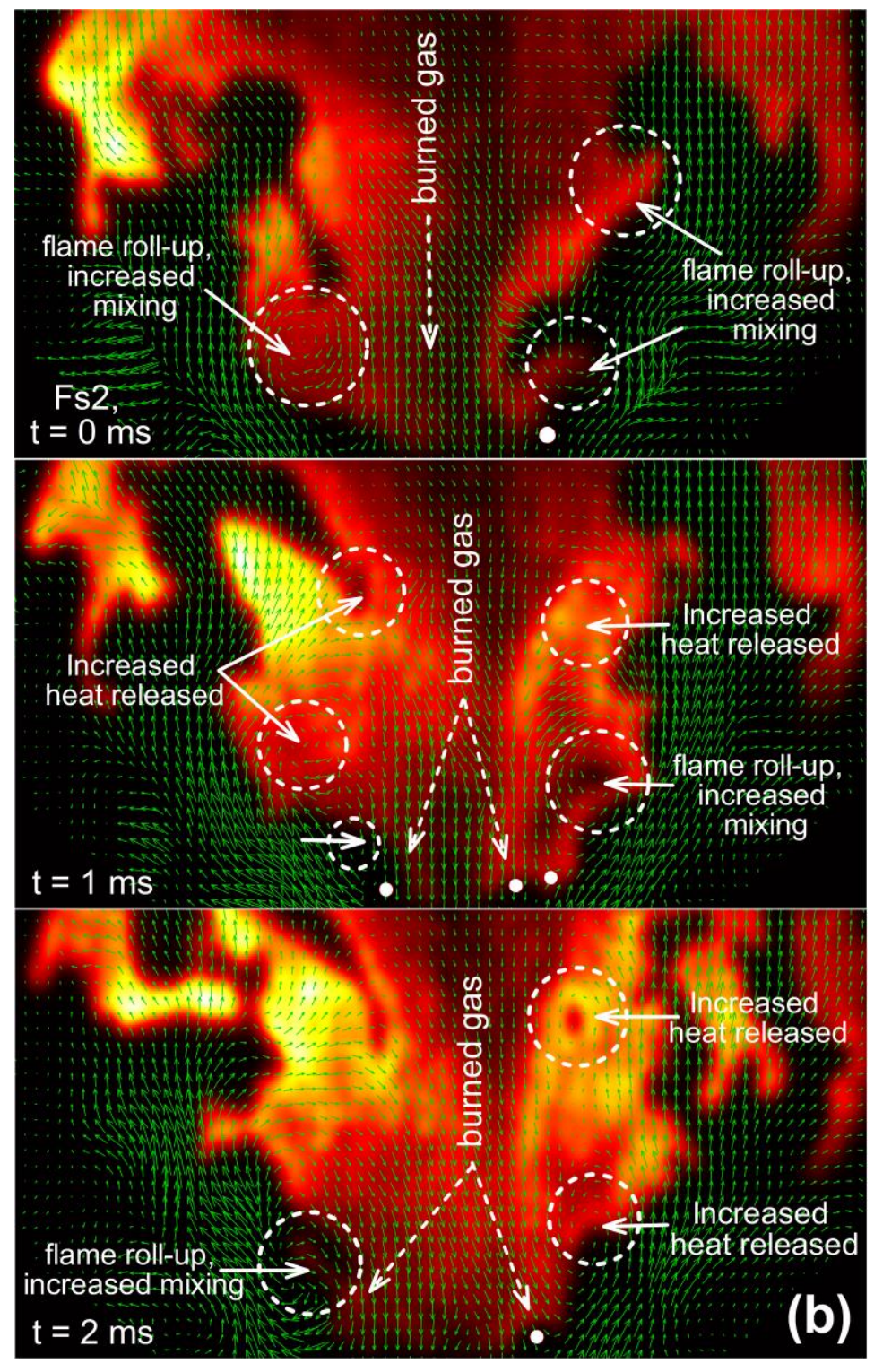

Fig. 9. Continued: (b) the simultaneous OH-PLIF/PIV for the frames at $\mathrm{t}=0,1$, and $2 \mathrm{~ms}$ of the early flame region.

The instantaneous OH-PLIF images of flame Fs2 illustrated in Fig. 9a shows two regions of high $\mathrm{OH}$ signal. The first region shows a $\mathrm{V}$-shape, starting from the burner exit to $\mathrm{Z} \approx 25$ $\mathrm{mm}$ downstream and expanding radially to $\pm 20 \mathrm{~mm}$. The second high $\mathrm{OH}$ region emerges from the end of the early $\mathrm{V}$-shape part and forms an M-shape, which extends radially to \pm 30 
$\mathrm{mm}$. The spatial position and structure of $\mathrm{OH}$ distribution concerning the flow field for the three of these OH-PLIF frames are displayed Fig. 9b. The majority of the $\mathrm{OH}$ is located in the ISL and IRZ with the V-shape region. It exhibits corrugated flame fronts at the vicinity of the ISL with a high $\mathrm{OH}$ at the boundaries and a moderate uniform $\mathrm{OH}$ in the central area. The locations with maximum LIF intensity is seen in the shear layers are indicative of superequilibrium concentrations. However, the inner boundaries of the recirculation zones are characterized by medium $\mathrm{OH}$ intensities with a more homogeneous distribution. This is typical of equilibrium $\mathrm{OH}$, indicating hot exhaust gas. This further confirms that the heat release observed by the $\mathrm{OH}^{*}$ as shown in Fig. 3, mostly takes place along the ISL.

The close inspection of the V-shape region, indicates two flame structures are formed. The first zone where the reaction zone elongates along the ISL between the IRZ and the incoming jet flow, and, a second flame structure zone is located immediately downstream the burner exit nozzle; namely, the flame root, see Fig. 9a. The reaction zone propagates downstream along with the shear layer, track the progress of the two $\mathrm{OH}$ pockets (a) and (b) with the time. This phenomenon is further illustrated by studying the simultaneous OH-PIV measurements displayed in Fig. 9b. The flow field shows the downstream motion of the vortices along the ISL (each marked with an arrow and bounded by the dashed circle). At time $\mathrm{t}=0 \mathrm{~ms}$, it is noticed that the eddy on the left and right side shear layers roll up the reaction zones, and thereby leads to an increase in the flame surface area. This leads to an increased in mixing between the burned and unburned fresh mixture. Following the right hand lower vortex associated with the $\mathrm{OH}$ pocket (b) at $\mathrm{t}=0 \mathrm{~ms}$, the burned gas is deflected from the stagnation point (of the flame root) towards the right-hand side. In the following frames at $\mathrm{t}=1$ and $2 \mathrm{~ms}$, the deflected burned gas is further rolled up by the vortex zone, and this enlarges the boundary between the burned and unburned gas, this promotes ignition of the 
unburned gas. As indicated at $\mathrm{t}=2 \mathrm{~ms}$ of the $\mathrm{OH} / \mathrm{PIV}$ frame, subsequently the $\mathrm{OH}$ levels become more substantial and propagate with the movement of the vortex downstream.

On the other hand, the flame root located downstream the nozzle exit, as indicated clearly in $\mathrm{OH} / \mathrm{PIV}$ frame, is located around the instantaneous stagnation point where the fresh gas from the nozzle and the burned gas from the IRZ collide frontally. This frontal collision with the downstream burned gas from the IRZ leads to the efficient supply of heat and radicals to the fresh gas close to the burner exit, as clearly indicated in PIV/OH frame at $\mathrm{t}=1$ and $2 \mathrm{~ms}$. These observed two zones suggest that there is a mutual interlink between them. The flame root essentially requires the upstream flow of hot exhaust gas in the IRZ coming from the elongated and propagated $\mathrm{OH}$ zone along ISL. On the other hand, the flame root is a source of heat and radicals close to the upstream end of the reaction zone along the ISL and therefore contributes to the ignition of the fresh gas in the ISL. The upper M-shape flame has a relatively weak $\mathrm{OH}$ signal at the central part of the flame with a high signal from the branched of the M-shape. Due to the high axial velocity associated with the swirling jet stream these $\mathrm{OH}$ branches are located at the outer shear layer and propagate upstream, see the time sequences of the $\mathrm{OH}$ pocket (c), illustrated in Fig. 9a.

Figure 10 shows a time series of OH-PLIF for flame Fs6 (Fig. 10a) together with the simultaneous OH-PLIF/PIV of two frames at $\mathrm{t}=0$ and $1 \mathrm{~ms}$ (Fig. 10b). There is a weak $\mathrm{OH}$ signal at the central flame region, with a very thin $\mathrm{OH}$-layer close to the burner nozzle along the ISL. As illustrated by the simultaneous OH-PLIF/PIV, the flame root is extinguished, and the reaction zone along the ISL is advected downstream. In contrast to flame Fs2, where the flame root is active, the flame roll-up along with the ISL does not lead to the generation of reaction zone close to the central region of the burner exit due to the high strain rates associated with the IRZ. This is indicated by the nearly zero $\mathrm{OH}$ signal along the flame centerline. These results demonstrate that the heat and radicals supply by the flame root plays 
an essential role in flame stabilization. Although the formation of the vortices along the ISL (as shown by the flow field in Fig. 10b), the more substantial reversed momentum associated the IRZ leads to quenching and diluting the active radicals from the downstream in their way towards the flame upstream. Several distinct processes can finally extinguish the flame root, the entrainment of cold unburned gas into the IRZ, and higher strain rates close to the burner nozzle, and intermittent variation in the mixture fraction in the vicinity of the flame root.

For flame Fs1, as shown in Fig. 11, where the time sequence of the OH-PLIF images indicate that the flame acts as a lifted-jet diffusion flame, but with a growing fragmented flame kernel along with the central region. Due to the low swirl number, no IRZ is formed, and thus there is a low probability of vortices close to the burner exit. The flame starts to liftoff, see the simultaneous OH-PIV frames, in Fig. 11. The OH/PIV measurements, illustrated in Fig. 11b, show that the instantaneous flame base is anchored primarily in the low-velocity regions of the jet, with the axial and radial movement of the flame to meet this criterion. With no formation of an IRZ, less fuel-air mixing occurs in this flame within the early flame region, which leads to a relatively cold inner flame region close the burner exit. These results highlight the mutual influences of the flame root and the helical flame, where the existence of both of them is crucial to have high stability inverse swirling flame. In addition, this suggests more modifications may be available to have a highly stabilized, non-premixed swirl inverse flame through the injection of a premixed pilot fuel/air-flow into the center of the inlet flow. 


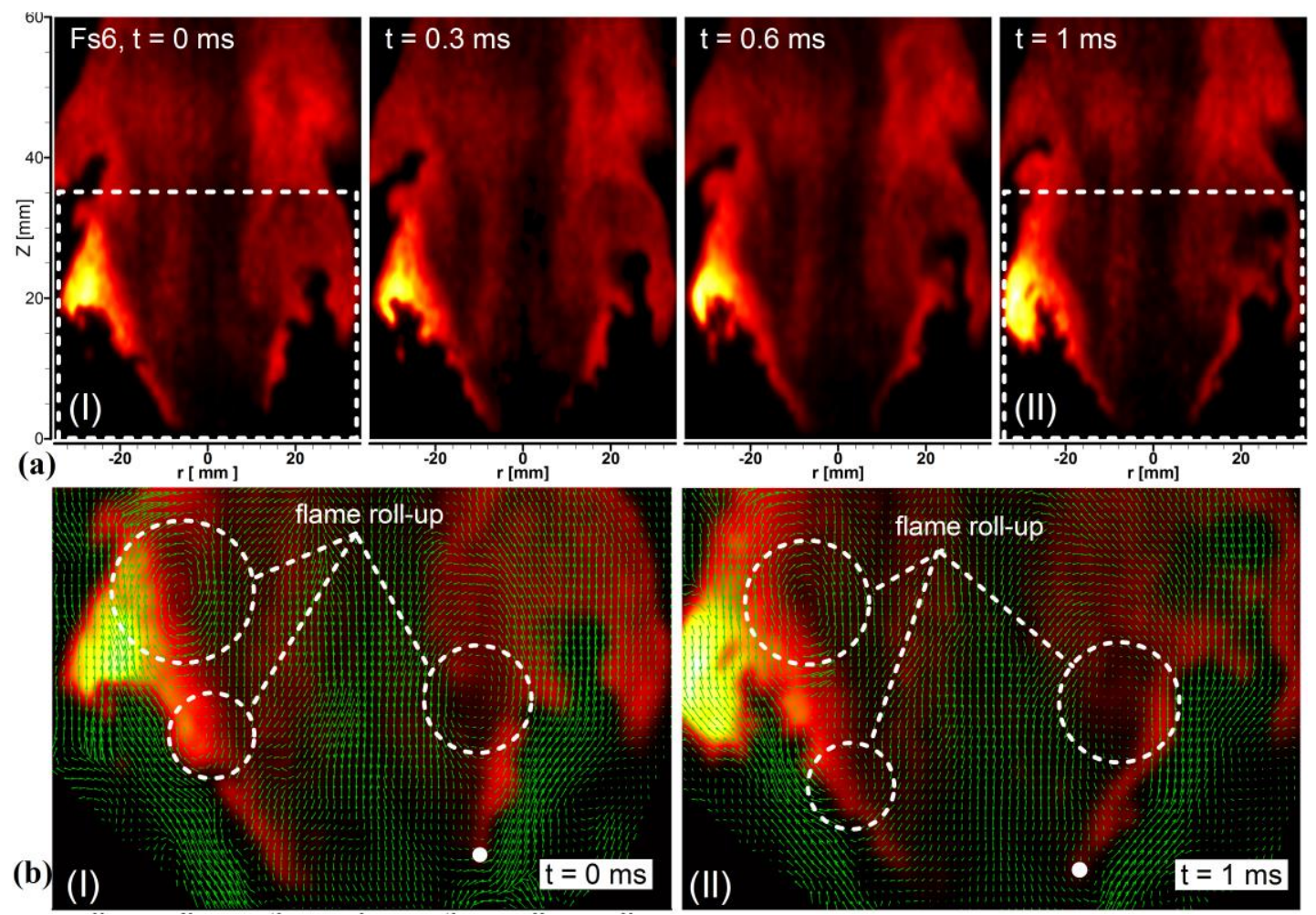

Fig. 10. (a) OH-PLIF time sequence of flame Fs6, and (b) the corresponding simultaneous $\mathrm{OH}-$ $\mathrm{PLIV} / \mathrm{PIV}$ at $\mathrm{t}=0$ and $1 \mathrm{~ms}$.

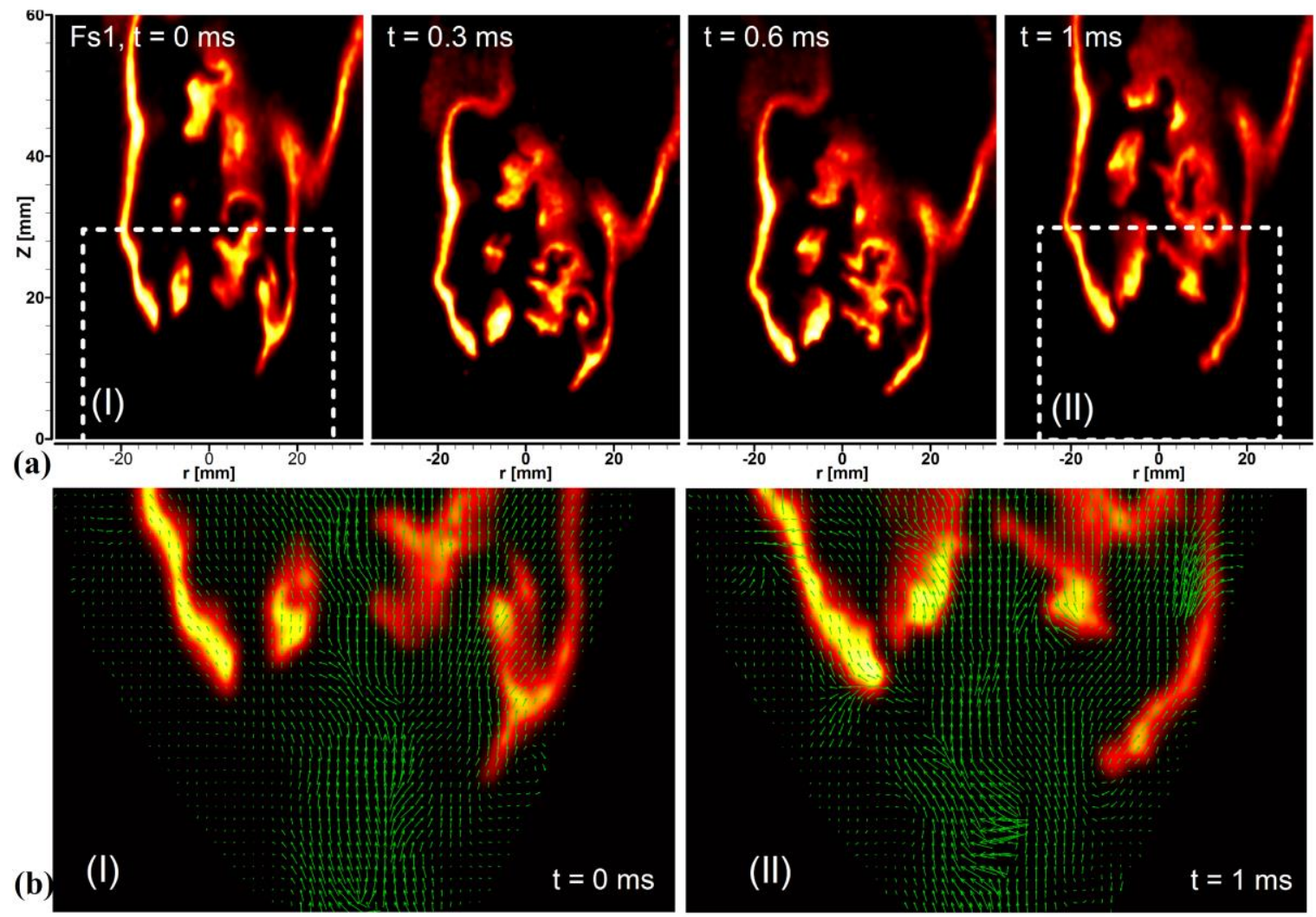

Fig. 11. (a) OH-PLIF time sequence of flame Fs1, and (b) the corresponding simultaneous $\mathrm{OH}-\mathrm{PLIV} / \mathrm{PIV}$ at $\mathrm{t}=0$ and $1 \mathrm{~ms}$. 


\section{Summary and conclusions}

The stability of an inverse non-premixed swirl flame was mapped. Under a fixed bulk air velocity and over a wide range of air swirl numbers, S, the fuel flow rate was gradually decreased until the extinction of the flame occurred. The most stable compact flame is achieved at critical swirl number, $S_{\text {cr. }}$ At $S<S_{c r}$, a lifted swirl jet-like flame is obtained. However, the compact swirl flame starts to lift off with the gradual increase in the swirl intensity. This is indicative of the complex flame/flow interactions inherent in swirl flames. To investigate these interactions, OH-PLIF measurements at $10 \mathrm{kHz}$ were acquired with simultaneous $\mathrm{OH}-\mathrm{PLIF} / \mathrm{PIV}$ at $1 \mathrm{kHz}$ on three different flames. One flame was at $\mathrm{S}_{\mathrm{cr}}$, while the other two flames were at higher or lower S, in the compact and lifted jet-like flame regions, respectively. For the flame at $\mathrm{S}_{\mathrm{cr}}$, the velocity and $\mathrm{OH}$ fields showed that there are two regions where the reactions take place. One of these regions has a conical shape and located along with the inner shear layer, ISL, between the inflow jet and the recirculation zone, IRZ. Due to the low strain rates in this region the ISL enhances flame stability. The other region is the flame root located near the burner nozzle around the instantaneous stagnation point, which is characterized by an opposed flow configuration of hot burned gas (formed downstream) and fresh fuel/air. With the gradual increase of the swirl, $S>S_{c r}$, the flame root is inherently destabilized due to the increased strain rates of the reversed flow. Eventually, the conical flame starts to lift-off, resulting in lower stability. At the low swirl number, there is no IRZ, and it is less probable that vortices formed along the shear layer can lead to greater fuel-air mixing, and the flame then acts as a pure jet flame. In this case, the flame is anchored along with the low- axial velocity shear layer. These results highlight the mutual dependence of the two flame zones, i.e., the flame root and the conical flame along the ISL. The flame root essentially requires the upstream flow of hot exhaust gases in the IRZ coming from the growing conical flame zone downstream. On the other hand, the flame root is a source of heat and radicals close to the upstream of the conical flame zone. These mutual 
interactions are responsible for the enhanced stability limits of swirling inverse jet diffusion

flames.

\section{References}

[1] F.J. Weinberg, The first half-million years of combustion research and today's burning problems, Prog. Energy Combust. Sci., 1 (1979) 17-31.

[2] A.M. Elbaz, W.L. Roberts, Flame structure of methane inverse diffusion flame, Exp. Therm. Fluid Sci., 56 (2014) 23-32.

[3] A.M. Elbaz, W.L. Roberts, Experimental study of the inverse diffusion flame using high repetition rate OH/acetone PLIF and PIV, Fuel, 165 (2016) 447-461.

[4] A.M. Elbaz, W.L. Roberts, Experimental characterization of methane inverse diffusion flame, Combust. Sci. Technol., 186 (2014) 1249-1272.

[5] A. Sobiesiak, J.C. Wenzell, Characteristics and structure of inverse flames of natural gas, Proc. Combust. Inst., 30 (2005) 743-749.

[6] L. Sze, C. Cheung, C. Leung, flame, Appearance, temperature, and NOx emission of two inverse diffusion flames with different port design, Combust. Flame, 144 (2006) 237-248.

[7] V. Hariharan, D.P. Mishra, Static Flame Stability of Circumferentially Arranged Fuel Port Inverse Jet NonPremixed Flame Burner, Combust. Sci. Technol., (2019) 1-27.

[8] F. Hunger, M.F. Zulkifli, B.A. Williams, F. Beyrau, C. Hasse, Comparative flame structure investigation of normal and inverse turbulent non-premixed oxy-fuel flames using experimentally recorded and numerically predicted Rayleigh and OH-PLIF signals, Proc. Combust. Inst., 36 (2017) 1713-1720.

[9] K.-T. Wu, R.H. Essenhigh, Mapping and structure of inverse diffusion flames of methane, Proc. Combust. Inst., 20 (1985) 1925-1932.

[10] C.R. Shaddix, T.C. Williams, Measurements of the velocity field in laminar ethylene inverse jet diffusion flames, Combust. Flame, 4 (2009) 942-945.

[11] A.M. Elbaz, H. Moneib, K. Shebil, W.L. Roberts, Low NOX-LPG staged combustion double swirl flames, Renewable Energy, 138 (2019) 303-315.

[12] A.M. Elbaz, W.L. Roberts, Investigation of the effects of quarl and initial conditions on swirling nonpremixed methane flames: Flow field, temperature, and species distributions, Fuel, 169 (2016) 120-134.

[13] H. Zhen, C. Cheung, C. Leung, H. Li, Thermal and heat transfer behaviors of an inverse diffusion flame with induced swirl, Fuel, 103 (2013) 212-219.

[14] H. Zhen, C. Leung, C. Cheung, Heat transfer from a turbulent swirling inverse diffusion flame to a flat surface, Int. J. Heat Mass Transfer, 52 (2009) 2740-2748.

[15] H. Zhen, C. Leung, C. Cheung, Thermal and emission characteristics of a turbulent swirling inverse diffusion flame, Int. J. Heat Mass Transfer, 53 (2010) 902-909.

[16] H. Zhen, C. Leung, C. Cheung, A comparison of the thermal, emission and heat transfer characteristics of swirl-stabilized premixed and inverse diffusion flames, Energy Conversion Management, 52 (2011) 1263-1271.

[17] H. Zhen, C. Leung, C. Cheung, Emission of impinging swirling and non-swirling inverse diffusion flames, Applied Energy, 88 (2011) 1629-1634.

[18] H. Zhen, C. Leung, C. Cheung, Combustion characteristics of a swirling inverse diffusion flame upon oxygen content variation, Applied Energy, 88 (2011) 2925-2933.

[19] H. Zhen, C. Leung, C. Cheung, A comparison of the thermal, emission and heat transfer characteristics of swirl-stabilized premixed and inverse diffusion flames, Energy Conversion Management

52 (2011) 1263-1271.

[20] X. Liu, A.M. Elbaz, C. Gong, X. Bai, H. Zheng, W.L. Roberts, Effect of burner geometry on swirl stabilized methane/air flames: A joint LES/OH-PLIF/PIV study, Fuel, 207 (2017) 533-546.

[21] A.M. Elbaz, S. Yu, X. Liu, X. Bai, I. Khesho, W.L. Roberts, An experimental/numerical investigation of the role of the quarl in enhancing the blowout limits of swirl-stabilized turbulent non-premixed flames, Fuel, 236 (2019) 1226-1242.

[22] A.M. Elbaz, Early structure of LPG partially premixed conically stabilized flames, Exp. Therm. Fluid Sci., 44 (2013) 583-591.

[23] M.S. Mansour, A. Elbaz, M.J.E.T. Samy, The stabilization mechanism of highly stabilized partially premixed flames in a concentric flow conical nozzle burner, Exp. Therm. Fluid Sci., 43 (2012) 55-62.

[24] A.M. Elbaz, M. Zayed, M. Samy, W.L. Roberts, M.S. Mansour, The flow field structure of highly stabilized partially premixed flames in a concentric flow conical nozzle burner with coflow, Exp. Therm. Fluid Sci., 73 (2016) 2-9. 
[25] M.S. Mansour, A.M. Elbaz, M. Zayed, Flame kernel generation and propagation in turbulent partially premixed hydrocarbon jet, Combust. Sci. Technol., 186 (2014) 698-711.

[26] T. Claypole, N. Syred, Effect of swirl burner aerodynamics on NOx formation, Proc. Combut. Inst., 18 (1981) 81-89.

[27] YI. Hardalupas, M. Orain, Local Measurements of the time-dependent heat release rate and equivalence ratio using chemiluminescent emission from a flame, Combust. Flame, 139 (2004) 188-207.

[28] M. Stöhr, I. Boxx, C. Carter, W. Meier, Dynamics of lean blowout of a swirl-stabilized flame in a gas turbine model combustor, Proc. Combust. Inst., 33 (2011) 2953-2960.

[29] R Sadanandan, M. Stöhr, W. Meier, Simultaneous OH-PLIF and PIV measurements in a gas turbine model combustor, Applied Physisc B, 90 (2008) 609-618. 